\title{
Electron Diffraction study of Layer Structures in La-Mg-Ni Hydrogen Absorption Alloys
}

\author{
Yasuyuki Kitano ${ }^{1,3}$, Tetsuya Ozaki ${ }^{1}$, Manabu Kanemoto ${ }^{2}$, Masao Komatsu ${ }^{3}$, \\ Shigeo Tanase ${ }^{1}$ and Tetsuo Sakai ${ }^{1}$ \\ ${ }^{1}$ National Institute of Advanced Industrial Science and Technology, Ikeda 563-8577, Japan \\ ${ }^{2}$ GS Yuasa Co., Kyoto 601-8520, Japan \\ ${ }^{3}$ Hiroshima Institute of Technology, Hiroshima 731-5193, Japan
}

\begin{abstract}
In order to improve the hydrogen absorption properties of the layer structure appearing at the nickel rich side of La-Mg-Ni alloy, appropriate amounts of $\mathrm{Ni}$ were replaced by some elemental atoms, $\mathrm{Mn}$, Co and $\mathrm{Al}$. The $\mathrm{La}_{0.8} \mathrm{Mg}_{0.2} \mathrm{Ni}_{3.4-x} \mathrm{Co}_{0.3}\left(\mathrm{MnAl}_{x}(0<x<0.4)\right.$ alloy system was synthesized and crystal structures were investigated by electron microscopy. The atomic concentration ratio of (LaMg) to the other atoms was $3.7 \mathrm{in}$ this system. The $\mathrm{Pr}_{5} \mathrm{Co}_{19}$-type structure $\left(5: 19 \mathrm{H}\right.$-structure) and the $\mathrm{Ce}_{5} \mathrm{Co}_{19}$-type structure (5:19R-structure) were found in this alloy system. Electron micrographs of these structures are presented in this paper. Structure analysis of the complex layer structure is demonstrated by careful observation of the electron diffraction pattern. [doi:10.2320/matertrans.MBW200631]
\end{abstract}

(Received December 8, 2006; Accepted May 10, 2007; Published July 11, 2007)

Keywords: crystal structure, nickel rich side of lantern-magnesium-nickel-cobalt-manganese-aluminum alloys, electron microscopy, hydrogen absorption

\section{Introduction}

Various layer structures were reported in the nickel rich side of La-Ni alloy system, such as $\mathrm{LaNi}_{2}$ (meta-stable), $\mathrm{LaNi}_{3}, \mathrm{La}_{2} \mathrm{Ni}_{7}, \mathrm{La}_{5} \mathrm{Ni}_{19}$ and $\mathrm{LaNi}_{5}{ }^{1-3)}$ Crystal structures homologous to these alloys were originally studied in the R-T alloy systems $(\mathrm{R}=$ Rare earth metals or Yttrium and $\mathrm{T}=$ Iron, Cobalt or Nickel) by Kahn. ${ }^{4-6)}$ Kahn pointed out that the structures could be regarded as a mixture of two structures, the Laves phase and the $\mathrm{CaCu}_{5}$ structure. Eelectron microscopic studies were performed by Komura et al. for the SmCo and Sm-Ni alloys. ${ }^{7-11)}$ They reported that several complex structures coexisted in the same grain. The microstructures were interpreted by using the term 'intergrowth'. They also established by high resolution electron microscopy the attribution of the images in the crystal structures.

Recently, it has been reported that an appropriate amount of $\mathrm{Mg}$ addition to the La-Ni alloy not only maintains the crystal structures but also gives an enhanced hydrogen storage capacity. ${ }^{12-17)}$ In order to improve farther the hydrogen properties of the $\mathrm{La}-\mathrm{Mg}-\mathrm{Ni}$ alloys, appropriate amounts of $\mathrm{Ni}$ were replaced by some elemental atoms, $\mathrm{Mn}$, Co and Al. Then alloys of $\mathrm{La}_{0.8} \mathrm{Mg}_{0.2} \mathrm{Ni}_{3.4-x} \mathrm{Co}_{0.3}(\mathrm{MnAl})_{x}(0<x<$ $0.4)$ were synthesized and studied. X-ray crystallographic analysis as well as electron microscopic analysis were carried out and reported partially in the previous papers. ${ }^{18,19)}$ In this paper, we present electron micrographs for the $\operatorname{Pr}_{5} \mathrm{Co}_{19}$-type structure $\left(5: 19 \mathrm{H}\right.$-structure) and the $\mathrm{Ce}_{5} \mathrm{Co}_{19}$-type structure (5:19R-structure) which were found in the alloy system, and also demonstrate that a complex layer structure can be analyzed by careful observation of the electron diffraction pattern.

\section{Experimental}

Alloy ingots were synthesized by induction-melting and were appropriately annealed in the same way as reported in the previous papers. ${ }^{18,19)}$ Specimens suitable for electron microscopy were prepared using a mechanical polishing machine and an ion thinning instrument. Two electron microscopes were employed, JEM2010 in Hiroshima Institute of Technology, and H9000 at the Research Center for Ultrahigh Voltage Electron Microscopy, Osaka University. The former was used for precise observation of the electron diffraction pattern and the latter was used for the high resolution imaging. Both were performed with the [010] incidence of the hexagonal indices of the crystal. In this direction, the structural information could be effectively collected.

\section{Results and Discussion}

3.1 Layer structures in the $\mathrm{La}_{0.8} \mathrm{Mg}_{0.2} \mathrm{Ni}_{3.4-x} \mathrm{Co}_{0.3}-$ $(\mathrm{MnAl})_{x}(0<x<0.4)$ alloys and description of the crystal structure

As mentioned in the previous section, several intermetallic compounds, have been reported in the $\mathrm{La}-\mathrm{Ni}$ or $\mathrm{La}-\mathrm{Mg}-\mathrm{Ni}$ alloy system, such as $\mathrm{LaNi}_{2}$ (meta-stable, cF24-MgCu 2 , cubic Laves phase: $\left.{ }^{1,2,16}\right), \mathrm{LaMgNi}_{4}\left(\mathrm{cF} 24-\mathrm{MgSnCu}_{4}\right.$, cubic ordered Laves phase $\left.{ }^{14,15)}\right), \mathrm{LaNi}_{3}\left(\mathrm{hR} 36-\mathrm{PuNi}_{3}, 1: 3 \mathrm{R}\right.$-structure $\left.{ }^{1)}\right)$, $\mathrm{LaMg}_{2} \mathrm{Ni}_{9}$ (hR36-PuNi $3,1: 3 \mathrm{R}$-structure ${ }^{13)}$ ), $\mathrm{La}_{2} \mathrm{Ni}_{7}$ (hP36$\mathrm{Ce}_{2} \mathrm{Ni}_{7}, 2: 7 \mathrm{H}$-structure ${ }^{1)}$ ), $\mathrm{La}_{5} \mathrm{Ni}_{19}$ (hR72-Ce $\mathrm{Co}_{19}$, 5:19Rstructure ${ }^{3)}$ ), $\mathrm{La}_{0.7} \mathrm{Mg}_{0.3} \mathrm{Ni}_{.2 .5} \mathrm{Co}_{0.5} \quad\left(\mathrm{hR} 72-\mathrm{Ce}_{5} \mathrm{Co}_{19}\right.$, 5:19Rstructure ${ }^{17)}$ ), $\mathrm{La}_{0.7} \mathrm{Mg}_{0.25} \mathrm{Ni}_{3.0} \mathrm{Co}_{0.5}$ (hP48- $\mathrm{Pr}_{5} \mathrm{Co}_{19}, 5: 19 \mathrm{H}-$ structure $\left.{ }^{17)}\right)$, and $\mathrm{LaNi}_{5}\left(\mathrm{hP6}-\mathrm{CaCu}_{5}{ }^{1)}\right)$.

In the La-Mg-Ni alloy system studied here, the existence of 1:3R-, 2:7H-, 5:19R-, 5:19H- and 1:4R-structures has been confirmed by X-ray diffraction analysis, and it has also been confirmed that $\mathrm{La}$ or $\mathrm{Mg}$ atoms occupy the site for large atoms and the $\mathrm{Ni}$ atoms occupy the site for small atoms. ${ }^{18,19)}$

The crystal structure of the Laves phase or the $\mathrm{CaCu}_{5}$ $\left(\mathrm{LaNi}_{5}\right)$ structure are known as closed pack structures of spheres with different radii. Atomic layers forming these structures are illustrated in Fig. 1. The hexagonal system 

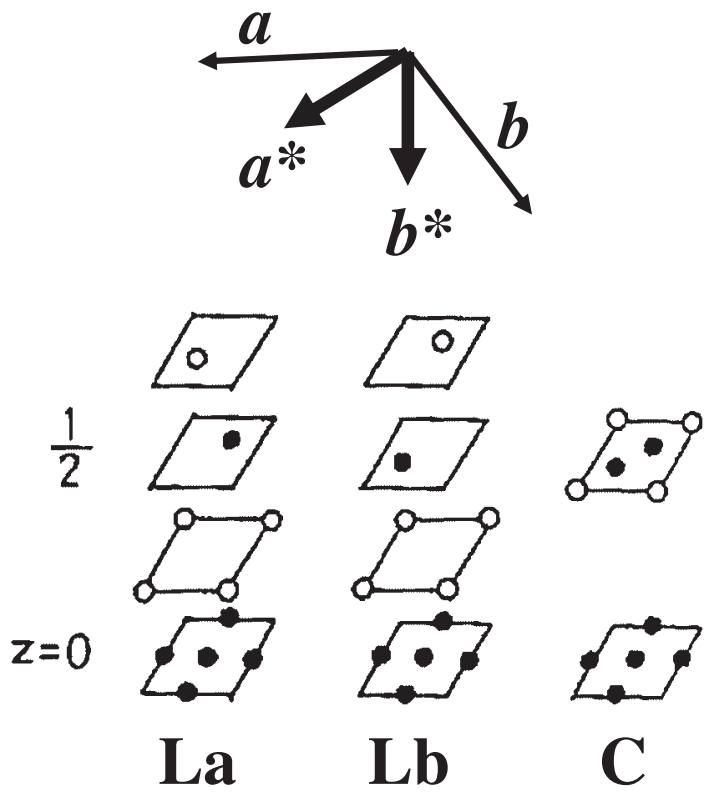

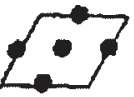

C

Fig. 1 Hexagonal setting assumed in this paper at the top, and drawings of the layer-units: the Laves phase, $\mathbf{L a} / \mathbf{L b}$, and the $\mathrm{CaCu}_{5}\left(\mathrm{LaNi}_{5}\right)$ structure, C. The thickness of the layer-unit is assumed to be equal for both, 0.404 $\mathrm{nm}$. When the layer-unit $\mathbf{L a}$ is followed by the layer-unit $\mathbf{C}$ (above), there is a shift $[-2 / 3-1 / 30]$. When the layer-unit $\mathbf{L b}$ is followed by the layerunit $\mathbf{C}$ (above), there is a shift $[2 / 31 / 30]$. When the layer-unit $\mathbf{C}$ is followed by one of the layer-units, $\mathbf{L a}, \mathbf{L b}$ and $\mathbf{C}$, there is no shift.

assumed in this paper is also included. Here $\mathbf{L a}$ or $\mathbf{L b}$ is a layer-unit of the Laves phase and $\mathbf{C}$ is a layer-unit of the $\mathrm{CaCu}_{5}\left(\mathrm{LaNi}_{5}\right)$ structure. As seen in Fig. 1, the layer-unit $\mathbf{L a} / \mathbf{L b}$ is composed of four atomic layers and contains 2 large and 4 small atoms, while the layer-unit $\mathbf{C}$ is composed of two atomic layers and contains 1 large and 5 small atoms. The total number of atoms included in a layer-unit is 6 for both Laves phase and $\mathrm{CaCu}_{5}$. The thickness of the layer-unit is almost the same for both structures. In this paper we will use the value $0.404 \mathrm{~nm}$ for the thickness, which was obtained by X-ray analysis. ${ }^{18,19)}$

In Fig. 2, an example of the TEM image is presented for the $5: 19 \mathrm{H}$ structure together with a projection of the crystal structure. The incidence of the electron beam is [010]. A part of the image is magnified and inserted at the top right corner of Fig. 2. Attribution of the image in relation to the structure has been already established. ${ }^{7-11)}$ Horizontal arrays of clear white dots correspond to one C-type layer-unit, and horizontal arrays of dull white dots in a dark line correspond to one $\mathbf{L a} / \mathbf{L b}$-type layer-unit. Three $\mathbf{C}$-type layer-units and one La/Lb-type layer-unit form a repeat-block in the $5: 19 \mathrm{H}$ structure. Positional correspondence of the image to the projection is indicated by three arrows. The relative shift between two repeat-blocks can be recognized negative or positive in the $[2 / 31 / 30]$ direction. In order to show the shift, a vertical line is drawn in the magnified image.

The crystal structure is built up by stacking repeat-blocks, each of which is formed by $\boldsymbol{m} \mathbf{C}$-type layer-units together with one $\mathbf{L a} / \mathbf{L b}$-type layer-unit at the top. A repeat-block stacks on the top of the La-type layer-unit with a shift of $[-2 / 3-1 / 30]$, while a repeat-block stacks on the top of the Lb-type layer-unit with a shift of $[2 / 31 / 30]$. The crystal

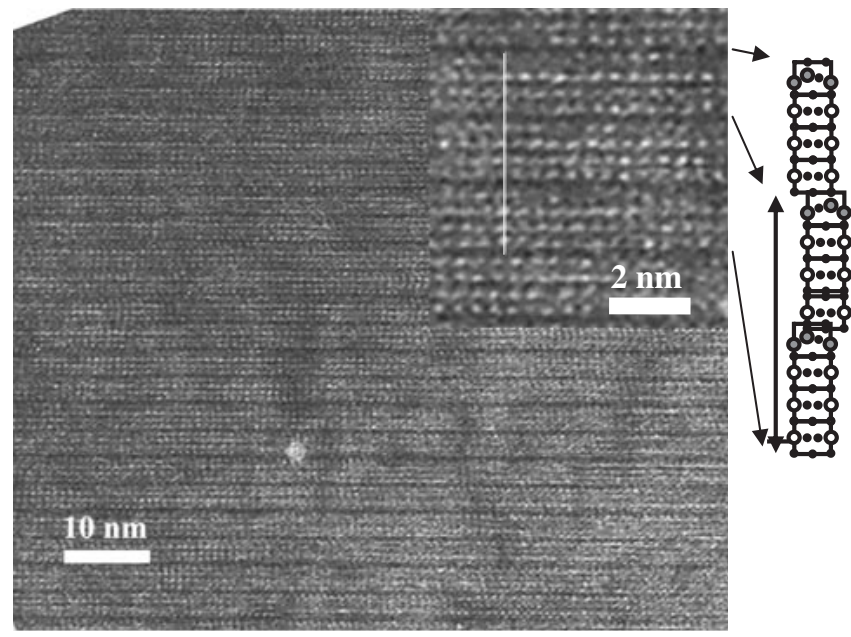

Fig. 2 TEM image of the $5: 19 \mathrm{H}$ structure found in the $\mathrm{La}_{0.8} \mathrm{Mg}_{0.2}$ $\mathrm{Ni}_{3.4-x} \mathrm{Co}_{0.3}(\mathrm{MnAl})_{x}(0<x<0.4)$ alloy compounds, and the corresponding projection. The incidence of the electron beam is [010]. A magnified image is inserted at the top right corner. Positional correspondence between the magnified image and the projection is indicated by three arrows. Here, we should note that, for the simplicity of the drawing, the projection is not a whole projection but only a half. The other half has been omitted in the figure. The omitted one is the same as the figure drawn but for a shift of [1 1/2 0].

structures discussed in this paper are summarized in Table 1. In the second column is presented the number of $\mathbf{L a / L b}$-type layer-units in a repeat-block, the numerals being singular for all cases. In the third column is presented the number of $\mathbf{C}$ type layer-units in a repeat-block and the numeral increases regularly depending on the crystal structure. This number will be denoted by $\boldsymbol{m}$ in this paper. The numbers ratio of large and small atoms is presented in the 4 th and 5th column. Type of the structure is given in the 6th column using an appropriate designation. Here, the symbol $\mathrm{H}$ means the hexagonal structure and $\mathrm{R}$ the rhombohedral one described by Ramsdell. ${ }^{20)}$ At the bottom of Table 1, the general form for the structure is presented using the parameter $\boldsymbol{m}$. Here we note that in large-scale analysis the value of $\boldsymbol{m}$ is not necessarily restricted to integers for complex crystal structures.

The electron diffraction pattern of the [010] zone axis is characterized by the distance between two spots along the $\mathrm{c}^{*}$ direction. Electron diffraction patterns for the alloys studied here are given in Fig. 3(a) and Fig. 3(b) for 5:19H and 5:19R structures, respectively. Indices in the reciprocal space are given in the hexagonal system. The distance between two reciprocal points, 000 and 001 corresponds to the inverse of the thickness of one $\mathbf{L a} / \mathbf{L b}$ - or $\mathbf{C}$-type layer-unit in Fig. 1, being approximately equal to $2.5 \mathrm{~nm}^{-1}$. A repeat-block for the $5: 19 \mathrm{H}$ structure is made of four layer-units in total, such as three C-type layer-units and one $\mathbf{L a} / \mathbf{L b}$-type layer-unit, and is the same as that for the 5:19R structure. Since repeatblocks for both consist of four layer-units, diffraction spots appear at a fourth of the distance from 000 to 001 . The stacking of repeat-blocks is different between the two structures. The unit cell size in the c direction for the hexagonal structure is twice of the repeat-block for the 5:19R structure, and therefore twice as many diffraction spots appear. 
Table 1 Crystal structures found in the Ni-rich side of the La-Ni or La-Mg-Ni system. The number of the layer-units, types $\mathbf{L a} / \mathbf{L b}$ and $\mathbf{C}$, in a repeat-block is given in the 2 nd and 3 rd column, respectively. The value $\boldsymbol{m}$ represents the latter. The normalized distance $\boldsymbol{t}$ between two diffraction spots along the $\mathrm{c}^{*}$ direction in the diffraction pattern of [010] zone axis is summarized in the last column. In the bottom row, the general form of the crystal structure is presented. The relation between two characteristic parameters, $\boldsymbol{m}$ and $\boldsymbol{t}$, are given in the last cell of the table. In this case, the value of $\boldsymbol{m}$ is not always restricted to integers.

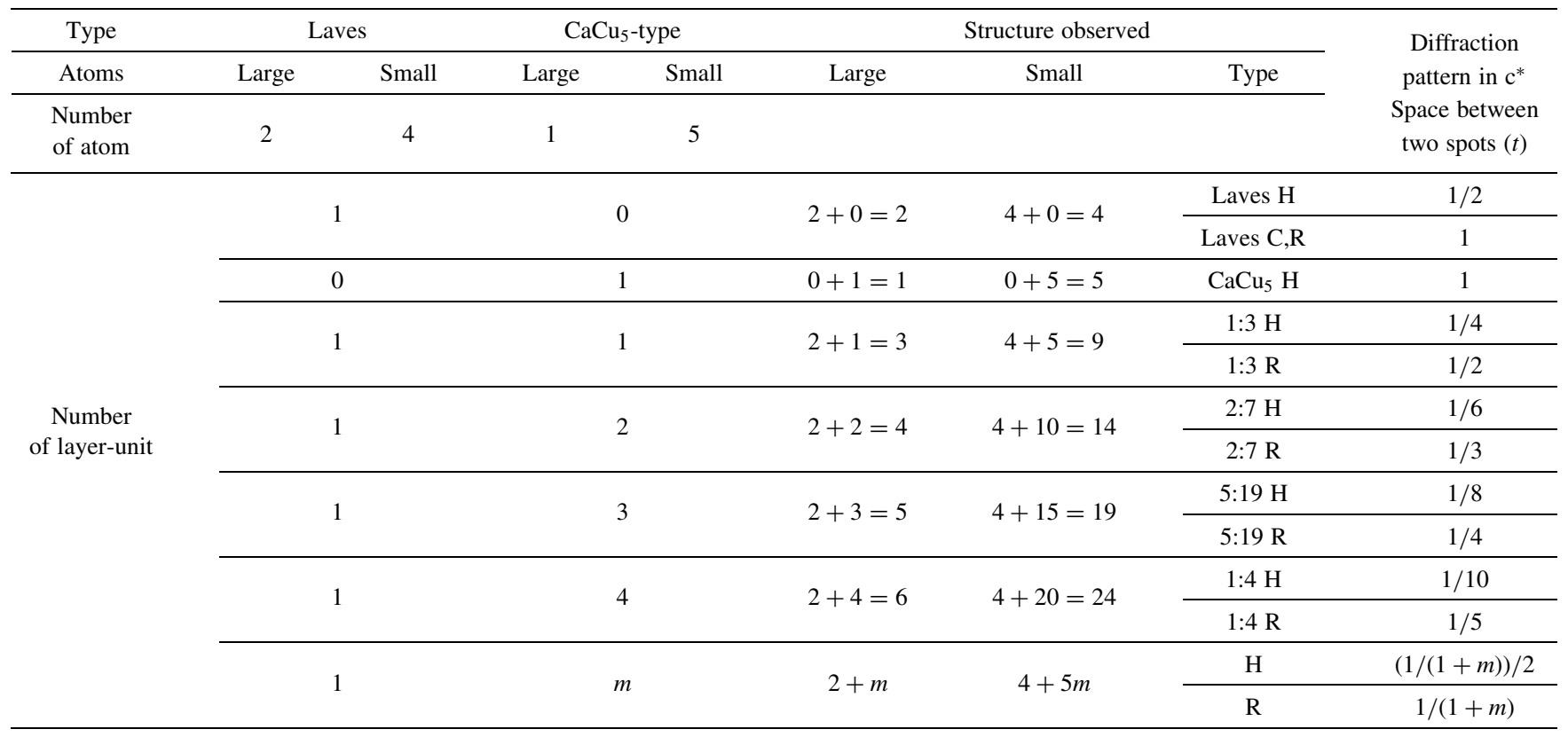
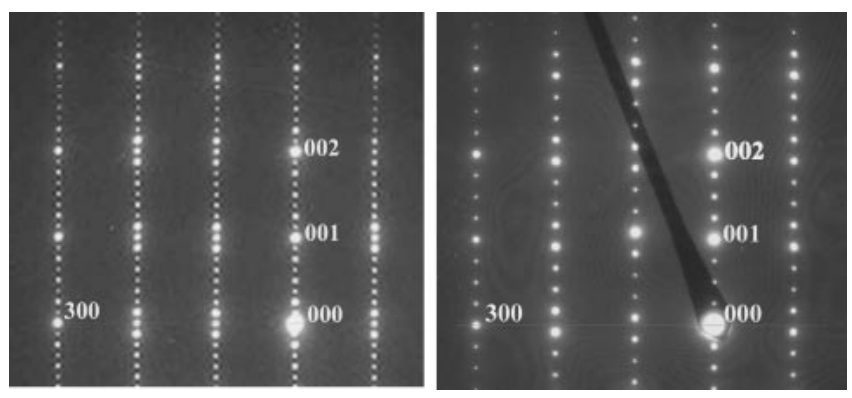

Fig. 3 Electron diffraction patterns found in the $\mathrm{La}_{0.8} \mathrm{Mg}_{0.2} \mathrm{Ni}_{3.4-x} \mathrm{Co}_{0.3}$ $(\mathrm{MnAl})_{x}(0<x<0.4)$ alloy compounds, (a) $\mathrm{Pr}_{5} \mathrm{Co}_{19}$ type $(5: 19 \mathrm{H}$ structure), (b) $\mathrm{Ce}_{5} \mathrm{Co}_{19}$ type (5:19R structure). Indices in the reciprocal space are given in the hexagonal system. Distance between two reciprocal points, 000 and 001 corresponds to the inverse of the thickness of one layer-unit, $\mathbf{L a} / \mathbf{L b}$ or $\mathbf{C}$ of Fig. 1, being approximately equal to $2.5 \mathrm{~nm}^{-1}$. Diffraction spots, 100 and 200, are visible for the hexagonal structure, but not for the rhombohedral structure.

In this paper, we define a parameter $t$ as a normalized distance between two diffraction spots along the $\mathrm{c}^{*}$ direction. In the last column of Table 1 , the $t$-value is summarized for the respective structures. At the lower right-hand corner cell, relations between two parameters, $\boldsymbol{m}$ and $\boldsymbol{t}$, are presented. The equations are as follows:

$$
\boldsymbol{t}=1 / 2(1+\boldsymbol{m}), \text { and then } \boldsymbol{m}=1 / 2 \boldsymbol{t}-1,
$$

for hexagonal,

and

$$
\begin{array}{r}
\boldsymbol{t}=1 /(1+m), \text { and then } \boldsymbol{m}=1 / \boldsymbol{t}-1, \\
\text { for rhomohedral, }
\end{array}
$$

where the parameter $t$ is the distance between two spots in c* direction in the diffraction pattern of the [010] zone axis, and

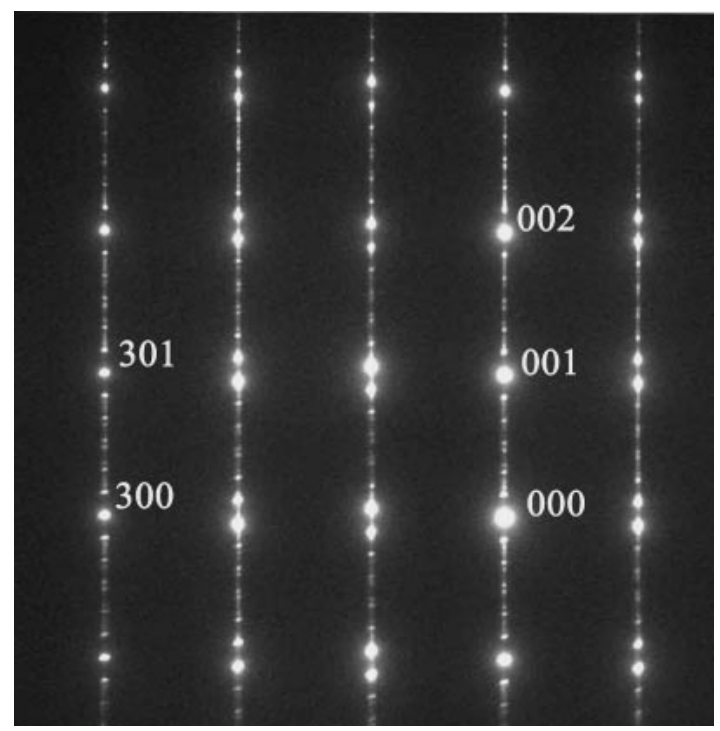

Fig. 4 Electron diffraction pattern of unknown structure. Zone axis is [010] in the hexagonal system. The diffraction spots align along the $\mathrm{c}^{*}$ direction not with a constant spacing, that is, the spots are incommensurate.

the parameter $\boldsymbol{m}$ is the number of $\mathbf{C}$-type layer-units in one repeat-block. In this equation, the value of $\boldsymbol{m}$ is not restricted to integers. It should be noted that in the diffraction pattern, as seen in Fig. 3, diffraction spots 100 and 200 appear for the hexagonal structure, but not for the rhombohedral structure.

\subsection{Electron crystallography for unknown layer struc- ture}

An electron diffraction pattern of unknown structure is presented in Fig. 4. The diffraction spots align along the $\mathrm{c}^{*}$ direction not with a constant spacing, that is, the spots are 


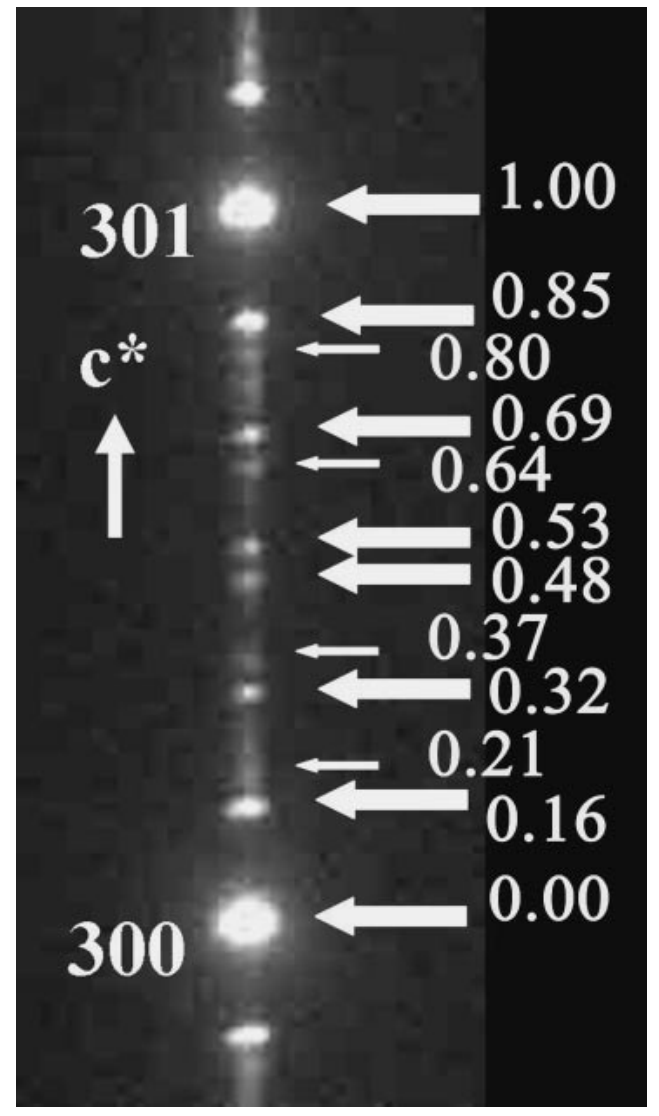

Fig. 5 Enlargement of Figure 4 between 300 and 301. The position of the diffraction spots is measured along the $c^{*}$ direction and spacing of the spots is found to be incommensurate, that is, $t=0.16$.

incommensurate. Diffuse streaks are also observed along the $\mathrm{c}^{*}$ direction. In the $\mathrm{a}^{*}$ direction, diffraction spots, 100 and 200 do not appear. These experimental facts suggest that the crystal structure of this area is rhombohedral, and the number of the $\mathbf{C}$-type layer-units might be different in each repeatblock. In that case, the parameter $\boldsymbol{m}$ would not be an integer.

An enlargement between 300 and 301 of Fig. 4 is presented in Fig. 5. In order to find out the precise value $t$ for the unknown structure, we measured the position of the diffraction spots between 300 and 301. The resulting positions of the diffraction spots are revealed in Fig. 5, and the period in the $\mathrm{c}^{*}$ direction is found to be $t=0.16$. Using the equation (2), the parameter $\boldsymbol{m}$ becomes 5.25. This does not correspond to any structure in Table 1.

It might be suggested that the structure is a mixture of two or more structures where the average value of the parameter $\boldsymbol{m}$ would become 5.25. As the simplest model, structure could be assumed to be a mixture of the two layer structures of $m=5$ and $m=6$. with a volume ratio 3 to 1 . Here the average value of the parameter $\boldsymbol{m}$ would be equal to $(5 \times$ $3+6 \times 1) / 4=5.25$.

A corresponding high resolution image is given in Fig. 6. The electron diffraction pattern Fig. 4 was made from a region approximately ten times larger than the area of Fig. 6. Since each layer-unit is resolved in each repeat-block in the image, we can see the layer structure directly. As mentioned in the previous section, the horizontal array of clear dots are attributed to the $\mathbf{C}$-type layer-unit and the horizontal arrays of

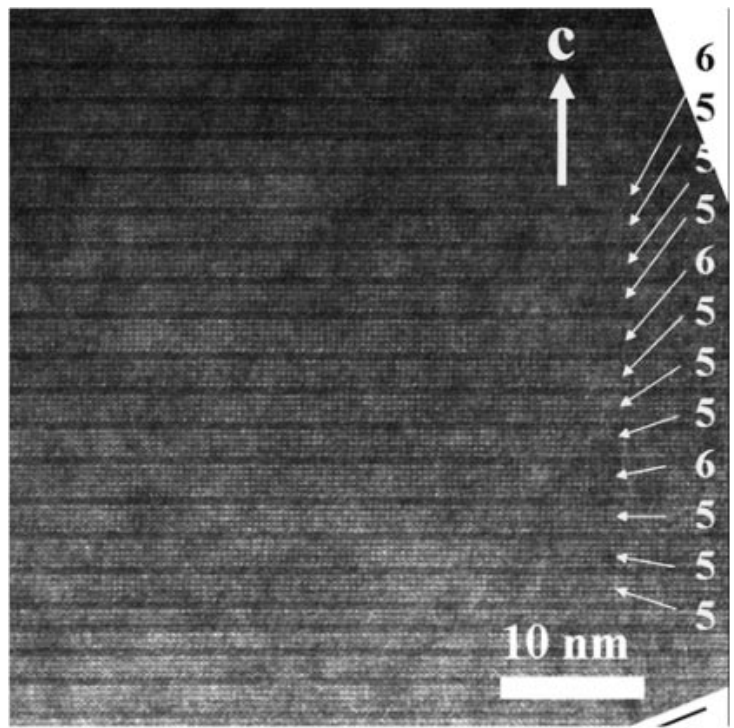

Fig. 6 High resolution image obtained from the area roughly corresponding to the diffraction pattern of Fig. 4 in the $\mathrm{La}_{0.8} \mathrm{Mg}_{0.2} \mathrm{Ni}_{3.4-x} \mathrm{Co}_{0.3}$ $(\mathrm{MnAl})_{x}(0<x<0.4)$ alloy compounds.

dull dots are attributed to the $\mathbf{L a / L b}$-type layer-units. Several types of repeat-blocks are found in the image.

Numerals inserted with an arrow in Fig. 6, present the number of the $\mathbf{C}$-type layer-units in each repeat-block in the central section of the image. Numerals other than 5 and 6 appeared above and below. The totally non-periodic structures can be observed in the image. Making an average for $\boldsymbol{m}$ at the main part of the image, we obtain that $\boldsymbol{m}$ is approximately equal to 5.25. This value is consistent with the $\boldsymbol{m}$-value obtained in the simplest model assumed from the electron diffraction pattern.

We have demonstrated that the parameter $\boldsymbol{m}$ (5.25) obtained from the high resolution images and the parameter $\boldsymbol{t}(0.16)$ measured in the electron diffraction pattern satisfy the equation (2) for the rhombohedral structure. In this way the complex crystal structure is plausibly analyzed from careful observation of the electron diffraction pattern.

\section{Acknowledgements}

A part of this work was supported by "Nanotechnology Support Project of the Ministry of Education, Culture, Sports, Science and Technology (MEXT), Japan” at the Research Center for Ultrahigh Voltage Electron Microscopy, Osaka University. Thanks to Professor H. Mori and Doctor K. Sakata of this center for the high resolution imaging by the H9000 electron microscope. The authors thank R\&D Support Project of Transmission Electron Microscopy in the Kansai centre of the National Institute of Advanced Industrial Science and Technology, for TEM specimen preparations of the alloys.

\section{REFERENCES}

1) P. Villars and L. D. Calvert: Pearson's Handbook of Crystallographic Data for Intermetallic Phases, vol. 4, second ed., ASM International, Materials Prak, Ohio, 1991. 
2) Binary Alloy Phase Diagram. ed. H. Okamoto. ASM International, Materials Park, OH, USA.

3) T. Yamamoto, H. Inui, M. Yamaguchi, K. Sato, S. Fujitani, I. Yonezu and K. Nishio: Acta Mater. 45 (1997) 5213-5221.

4) Y. Kahn: Acta Crystallography B30 (1970) 1533-1537.

5) Y. Kahn: Zeitschrift fuer Metallkunde 65 (1974) 489-495.

6) Y. Kahn: Phys. Stat. Sol. (a) 23 (1974) 425.

7) Y. Komura, S. Takeda and Y. Kitano: Science Report, RITU A29, Suppl. 1 (1981) 13-18.

8) S. Takeda, Y. Kitano and Y. Komura: J. Less-Common Metals 84 (1982) 317-325

9) S. Takeda and Y. Komura: Crystal research and technology 17 (1982) 1145-1150.

10) S. Takeda, H. Horikoshi and Y. Komura: J. Microscopy 129 (1983) 347-352.

11) H. Horikoshi, S. Takeda and Y. Komura: Jpn. J. Appl. Phys. 24 (1985) L210-L212.
12) H. Osterreich and H. Bitter: J. Less Common Met. 73 (1980) 339-344.

13) K. Kadir, T. Sakai and I. Uehara: J. Alloys Compd. 257 (1997) 115121.

14) K. Kadir, D. Noreus and I. Yamashita: J. Alloys Compd. 345 (2002) 140-143.

15) L. Guenee, V. Favre-Nicolin and K. Yvon: J. Alloys Compd. 348 (2003) 129-137.

16) S. De Negri, M. Giovannini and A. Saccone: J. Alloys and Compounds 397 (2005) 126-134

17) H. Hayakawa, E. Akiba, M. Gotoh and T. Kohno: Mater. Trans. 46 (2005) 1393

18) T. Ozaki, Y. Kitano, S. Tanase, T. Sakai, M. Kanemoto, T. Kakeya, M. Kuzuhara and M. Watada: ECS 208th Meeting, Abstract number 861, Los Angeles (2005).

19) T. Ozaki, M. Kanemoto, T. Kakeya, Y. Kitano, M. Kuzuhara, M. Watada, S. Tanase and T. Sakai: accepted by J. Alloys Compds.

20) L. S. Ramsdell: Amer. Mineralogist. 32 (1947) 64-82. 